\title{
Sequence-based identification of microbial contaminants in non-parenteral products
}

\author{
Rajapandi Senthilraj*, Ganduri Sathyanarayana Prasad, Kunchithapatham Janakiraman
}

\author{
Department of Pharmacy, Annamalai University, Annamalainagar, Chidambaram, India
}

\begin{abstract}
Phenotypic profiles for microbial identification are unusual for rare, slow-growing and fastidious microorganisms. In the last decade, as a result of the widespread use of PCR and DNA sequencing, 16S rRNA sequencing has played a pivotal role in the accurate identification of microorganisms and the discovery of novel isolates in microbiology laboratories. The $16 \mathrm{~S}$ rRNA region is universally distributed among microorganisms and is species-specific. Accordingly, the aim of our study was the genotypic identification of microorganisms isolated from non-parenteral pharmaceutical formulations. DNA was separated from five isolates obtained from the formulations. The target regions of the rRNA genes were amplified by PCR and sequenced using suitable primers. The sequence data were analyzed and aligned in the order of increasing genetic distance to relevant sequences against a library database to achieve an identity match. The DNA sequences of the phylogenetic tree results confirmed the identity of the isolates as Bacillus tequilensis, B. subtilis, Staphylococcus haemolyticus and B. amyloliqueficians. It can be concluded that $16 \mathrm{~S}$ rRNA sequence-based identification reduces the time by circumventing biochemical tests and also increases specificity and accuracy.
\end{abstract}

Uniterms Non-parenteral products/microbiological contamination. Microbes/identification/non-parenteral products. Phylogenetic analysis/microbial identification. 16S rRNA/use/microbial identification.

Os perfis fenotípicos para identificação microbiana são incomuns para micro-organismos raros, de crescimento lento e exigentes. Na última década, em resultado do uso generalizado de PCR e sequenciação de DNA, a sequenciação do rRNA $16 \mathrm{~S}$ tem desempenhado papel crucial na identificação precisa do micro-organismo e a descoberta de novos isolados em laboratórios de microbiologia. A região de rRNA 16S é universalmente distribuída entre micro-organismos e é espécie-específica. A genotipagem foi realizada sobre os organismos isolados a partir de formulações farmacêuticas não parenterais. O DNA foi separado dos cinco isolados obtidos a partir das formulações. As regiões alvo dos genes de rRNA foram amplificados por PCR e sequenciados utilizando os iniciadores adequados. Os dados dos sequência foram analisados e alinhados na ordem crescente de distância genética de sequências relevantes contra biblioteca de dados para obter a identidade. A sequência de DNA de árvores filogenéticas confirma a identidade dos isolados como Bacillus-tequilensis, B. subtilis, Staphylococcus haemolyticus e B. amyloliqueficians. Pode-se concluir identificação baseada na sequência do rRNA 16S reduz o tempo por evitar testes bioquímicos e também aumenta a especificidade e a precisão.

Unitermos: Produtos não-parenterais/contaminação microbiana. Micróbios/identificação/produtos nãoparenterais. Análise filogenética/identificação microbiana. 16S rRNA/use/ identificação microbiana.

\footnotetext{
*Correspondence: R. Senthilraj. Department of Pharmacy. Annamalai University. Annamalainagar-608002. Chidambaram, India. E-mail: senthilrajbt@gmail.com
} 


\section{INTRODUCTION}

Microbiological contamination is a serious problem, because it not only results in the spoilage of medicines but also causes infections, and hence, several classical culture methods have evolved to determine the microbiological quality of non-parenteral pharmaceutical products (Jasson et al., 2010; Rosa, Medina, Vivar, 1995). These protocols are time-consuming and labor-intensive to ensure the microbiological quality of non-parenteral products. The inevitable time delay associated with incubation often determines that microbiological quality assurance data are only of retrospective value. Modern pharmaceutical production and economic pressures can no longer accommodate this delay (Newby, 2000). In the past decade, nucleic acid sequencing methods have undergone tremendous advances (such as whole-genome sequencing as well as the determination of $16 \mathrm{~S}$ rRNA, 16S-23S rRNA, spacer and 23S rRNA sequences), which minimize the time needed for identification of microorganisms. 16S rRNA sequencing analysis is widely used, (Bansal, Meyer, 2002) and more useful in phylogenetic analysis compared to $16 \mathrm{~S}-23 \mathrm{~S}$ rRNA sequencing (Song et al., 2004) and also due to its rapidity, reliability, simplicity and reproducibility (Lane et al., 1985; Patel 2001; Easter 2003). This identification method not only confirms the microbial limits suggested in official pharmacopoeias but also demonstrates the presence of any other pathogens by virtue of its specificity (Rompre et al., 2002; Gee et al. 2003; Rhoads et al., 2012). Recent studies and micro sequence packages indicate the cost of analysis is low (Cook et al., 2003; Hall et al., 2003; Woo et al., 2003). $16 \mathrm{~S}$ rRNA sequencing is routinely used in the clinical laboratory but rarely used for microbial limits test for nonparenteral products. Hence, the aim of the present work was to evaluate $16 \mathrm{~S}$ rRNA sequence-based identification of microbial contaminants in non-parenteral products.

\section{MATERIAL AND METHODS}

(1) Sequencing Kit: ABI 3100 (Applied Biosystems) with Big Dye Terminator Kit v.3.1 (Applied Biosystems) (2) Universal primers: 27F ( 5'GAGT T T GATCATGGCTCAG3'), 1492 R (5'GGTTACCTTGTTACGACTT3') [for PCR] and 518F (5'CCAGCAGCCGCGGTAATACG3') and 800R (5'TACCAGGGTATCTAATCC3') [for 16S rRNA sequencing] were purchased from Macrogen. (3) Sequencer: ABI PRISM 3730XL Analyzer (96 capillary type) (3) PCR machine: MJ Research PTC-225 Peltier Thermal Cycler. All chemicals were purchased from
Hi-media \&Sigma Aldrich Mumbai. The non-parenteral products (syrup and tablets) were purchased from retail medical shops in TamilNadu.

\section{Preparation of the sample}

For isolation of specific organism present in the non parenteral products (Syrup, Tablet) six different media were used. All media including mannitol salt agar, MacConkey agar, xylose lysine deoxycholate agar, cetrimide agar, Columbia agar and Sabouraud dextrose agar were prepared according to the directions given on the label of the container and sterilized in an autoclave (USP35, 2012; IP, 2007). From syrup, microbial growth was observed on Sabouraud dextrose agar, and from tablets, microbial growth was observed on mannitol salt agar, Maconkey salt agar, xylose lysine deoxycholate agar and Sabouraud dextrose agar (Senthilraj, Prasad, Janakiraman, 2014). The microbial growth from syrup was coded as SYRSI2SAB and that from tablets as TABSIV1MAN, TABSIV2MAC, TABSIV3XYL and TABSIV4SAB, respectively. The isolated cultures were allowed to grow overnight in nutrient broth at $37^{\circ} \mathrm{C}$ for isolation of DNA.

\section{DNA Isolation}

The isolate was centrifuged at $5000 \mathrm{rpm}$, mixed with $10 \mu 1$ Tris-EDTA buffer ( $\mathrm{pH} 8.0)$, and centrifuged again until a pellet was obtained. The Tris-EDTA buffer and lysozyme were added to the microbial pellet and then incubated at $37^{\circ} \mathrm{C}$ for 30 minutes. The cells were lysed by the addition of $3 \mu \mathrm{L}$ of $10 \%$ sodium dodecyl sulfate and $3 \mu \mathrm{L}$ of proteinase $\mathrm{K}$ solution followed by a 15 -minute incubation at $37^{\circ} \mathrm{C}$. Subsequently, $1 \mu \mathrm{L}$ of $5 \mathrm{M}$ sodium chloride and $80 \mu \mathrm{L}$ of cetyltrimethylammonium bromide were added. The water phase was extracted with chloroform:isoamyl alcohol $(24: 1)(780 \mu \mathrm{L})$, and the mixture was centrifuged at $10,000 \mathrm{rpm}$. Isopropanol $(0.6$ $\mathrm{mL}$ ) was added to the supernatant and the mixture was again centrifuged at 10,000 rpm for $5 \mathrm{~min}$, after which the supernatant was removed. The ethanol washed, air-dried pellets were suspended in Tris-EDTA buffer and stored at $4{ }^{\circ} \mathrm{C}$ until they were used. (Nishiguchi et al., 2002; Vural, Ozgun, 2011). DNA was extracted using the QIAamp Tissue Kit (Loeffler et al., 1996).

\section{$16 S$ gene Amplification}

PCR for the amplification of the $16 \mathrm{~S}$ rRNA gene was carried out using universal primers $27 \mathrm{~F}$ (5'-AGAGTTTGATCATGGCTCAG-3') as forward and 
TABLE I - Percentage similarity of tested strains against representative species in BLAST search

\begin{tabular}{lccc}
\hline S. No. & Test strains & Representative species & Percentage similarity (BLAST) \\
\hline 1. & SYRSI2SAB & Bacillus tequilensis (NR 140919) & $99 \%$ \\
2. & TABSIV1MAN & Bacillus amyloliquefaciens (NR 116022) & $99 \%$ \\
3. & TABSIV2MAC & Bacilus subtilis (NR 112629) & $99 \%$ \\
4. & TABSIV3XYL & Sthaphylicoccus haemolyticus (NR 036955) & $99 \%$ \\
5. & TABSVI4SAB & Bacillus amyloliquefaciens (NR 112685) & $99 \%$ \\
\hline
\end{tabular}

1492R (5'-GGTTACCTTGTTACGACTT-3') as reverse primer. (Lane, 1991; Turner et al., 1999). Approximately 10 to $100 \mathrm{ng}$ of template were added to a reaction mix containing $20 \mathrm{mM}$ Tris- $\mathrm{HCl}(\mathrm{pH} \mathrm{8.4),} 50 \mathrm{mM}$ $\mathrm{KCl}, 1.5 \mathrm{mM} \mathrm{MgCl}, 2 \mu \mathrm{L}$ of each deoxynucleoside triphosphate, $0.4 \mu \mathrm{M}$ of each primer, and $2.5 \mathrm{U}$ Taq DNA polymerase to complete a final volume of $50 \mu \mathrm{L}$. The PCR conditions were: initial denaturation of $2 \mathrm{~min}$ at $94^{\circ} \mathrm{C}$, followed by 35 cycles of $1 \mathrm{~min}$ at $94{ }^{\circ} \mathrm{C}, 1.5 \mathrm{~min}$ at $55^{\circ} \mathrm{C}$ and $1 \mathrm{~min}$ at $72^{\circ} \mathrm{C}$, and a final extension at $72^{\circ} \mathrm{C}$ for $3 \mathrm{~min}$. The reaction product was visualized on a $1 \%$ agarose gel under UV light after ethidium bromide staining (Silva et al., 2013; Devereux, Wilkinson, 2004).

\section{$16 S$ rRNA gene sequencing and phylogenetic analysis}

The amplified PCR products of microbial gene fragments were purified and sequenced at MACROGEN sequencing company, Seoul, Korea using the automated sequencer ABI 3100 with Big Dye Terminator Kit v. 3.1. Primers 518F (5'CCAGCAGCCGCGGTAATACG3') and 800R (5'TACCAGGGTATCTAATCC3') were used for sequencing (Ghyselinck et al., 2013). The sequences thus obtained were compared with the NCBI database through BLAST searches (http://blast.ncbi.nlm.nih.gov/Blast.cgi). In this comparison, sequences of type strains most closely related to the sequences of the isolates were searched. The sequences were aligned with Clustal $\mathrm{W}$, and a phylogenetic tree was constructed from the evolutionary distances by the neighbor-joining method with the software MEGA (Nikunjkumar, 2012; Tamura et al., 2011).

\section{RESULTS AND DISCUSSION}

Isolate-1 from syrup (SYRSI2SAB) had a nucleotide sequence of $1425 \mathrm{bp}$ (Fig. 1). NCBI-BLAST search results showed the highest sequence similarity with Bacillus tequilensis belonging to the family Bacillaceae, and the accession number was NR 104919.

Isolates-2, $-3,-4$ and -5 from tablets (TABSIV1MAN,
TABSIV2MAC, TABSIV3XYL and TABSIV4SAB) had nucleotide sequences of 1600, 1882, 1590 and $1550 \mathrm{bp}$ and are shown in Figs. 3, 5, 7 and 9. The NCBI-BLAST search results for isolates- $2,-3,-4$ and -5 had highest sequence similarity with the following bacteria, whose accession numbers are given in parentheses: $B$. amyloliqueficians (NR116022), B. subtilis (NR112629), Staphylococcus haemolyticus (NR036955) and B. amyloliqueficians (NR104919). Thus, the BLAST search of Genbank for all isolates provided the percentage similarity between the microorganism tested and those detected in Genbank as shown in Table I.

Furthermore, the neighbor-joining tree based on 16S rRNA gene sequences was constructed to show the relationship between isolate- 1 and 12 representative species of the family Bacillaceae (Fig. 2). This result also confirmed the result of NCBI-BLAST for isolate-1. Likewise, for isolates-2, $-3,-4$ and -5 , the neighborjoining trees based on 16S rRNA gene sequences were constructed to show the relationship between the isolates and their representative species of the family Bacillaceae, and Staphylococcaceae, as shown in Figs. 4, 6, 8 and 10 . Thus, the above results showed that contaminants of non-parenteral pharmaceutical products belonged to Bacillus and Staphylococcus species and the names of the contaminants are given in Table II.

\section{CONCLUSION}

As discussed earlier, the universal primers $27 \mathrm{~F}$ and 1492R produced well-amplified 16S r RNA PCR products for all isolates. The nucleotide sequence obtained for all isolates was approximately $1500 \mathrm{bp}$ by using the universal sequencing primers $518 \mathrm{~F}$ and $800 \mathrm{R}$ (Ghyselinck et al., 2013) which are sufficient for NCBI-BLAST searches and phylogenetic analysis for the identification of unknown microorganisms.

The time for microbial identification was reduced by molecular-based PCR techniques when compared to conventional methods of detection. But PCR detects only the fixed target microorganism and it will not detect 


\begin{abstract}
AACTGGGGAACTTGAGTGCAGAGTAGGAGAA AGGAATTCCACGTGTAGCGGTGAAATGCGTAG AGATGTGGAGGAACACCAGTGGCGAAGGCGA CTCTCTGGTCTGTAACTGACGCTGAGGAGCGA AAGCGTGGGGAGCGAACAGGATTAGATACCCT GGTAGTCCACGCCGTAAACGATGAGTGCTAAG TGTTAGGGGGTTTCCGCCCCTTAGTGCTGCAGC TAACGCATTAAGCACTCCGCCTGGGGAGTACG GTCGCAAGACTGAAACTCAAAGGAATTGACGG GGGCCCGCACAAGCGGTGGAGCATGTGGTTTA ATTCGAAGCAACGCGAAGAACCTTACCAGGTC TTGACATCCTCTGACAATCCTAGAGATAGGAC GTCCCCTTCGGGGGCAGAGTGACAGGTGGTGC ATGGTTGTCGTCAGCTCGTGTCGTGAGATGTTC GGTTAAGTCCCGCAACGAGCGCAACCCTTGAT CTTAGTTGCCAGCATTCAGTTGGGCACTCTAAG GTGACTGCCGGTGACAAACCGGAGGAAGGTGG GGATGACGTCAAATCATCATGCCCCTTATGAC CTGGGCTACACACGTGCTACAATGGACAGAAC AAAGGGCAGCGAAACCGCGAGGTTAAGCCAA TCCCACAAATCTGTTCTCAGTTCGGATCGCAGT CTGCAACTCGACTGCGTGAAGCTGGAATCGCT AGTAATCGCGGATCAGCATGCACTGGTGTTCC TCCACATCTCTACGCATTTCACCGCTACACGTG GAATTCCACTCTCCTCTTCTGCACTCAAGTTCC CCAGTTTCCAATGACCCTCCCCGGTTGAGCCGG GGGCTTTCACATCAGACTTAAGAAACCGCCTG CGAGCCCTTTACGCCCAATAATTCCGGACAAC GCTTGCCACCTACGTATTACCGCGGCTGCTGGC ACGTAGTTAGCCGTGGCTTTCTGGTTAGGTACC GTCAAGGTACCGCCCTATTCGAACGGTACTTGT TCTTCCCTAACAACAGAGCTTTACGATCCGAA AACCTTCATCACTCACGCGGCGTTGCTCCGTCA GACTTTCGTCCATTGCGGAAGATTCCCTACTGC TGCCTCCCGTAGGAGTCTGGGCCGTGTCTCAGT CCCAGTGTGGCCGATCACCCTCTCAGGTCGGCT ACGCATCGTTGCCTTGGTGAGCCATTACCTCAC CAACTAGCTAATGCGCCGCGGGTCCATCTGTA AGTGGTAGCCGAAGCCACCTTTTATGTTTGAAC CATGCGGTTCAAACAACCATCCGGTATTAGCC CCGGTTTCCCGGAGTTATCCCAGTCTTACAGGC AGGTTACCCACGTGTTACTCACCCGTCCGCCGC TAACATCAGGGAGCAAGCTCCCATCTGTCCGC TCGACTTGCATGTATTAGGCACGCCGCCAGCG TT.
\end{abstract}

FIGURE 1 - The 16S rRNA sequence generated for isolate-1 had 1425 bases.

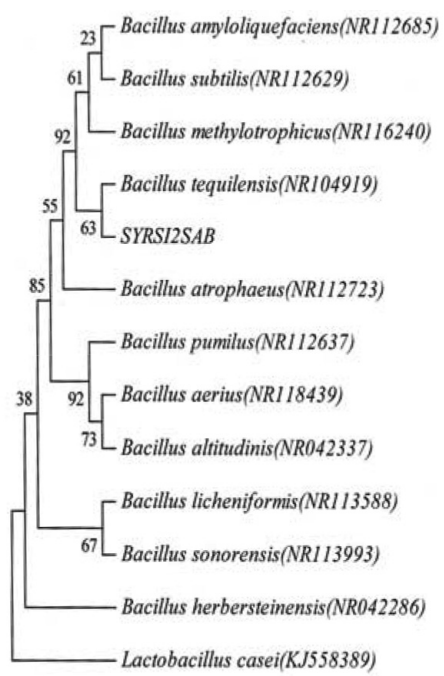

FIGURE 2 - Neighbor-joining tree based on 16S rRNA (1425) sequences showing the relationship between unknown isolate SYRSI1SAB and other closely related species of the genus Bacillus.
CTTAAGTCTGATGTGAAAGCCCCCGGCTCAAC CGGGGAGGGTCATTGGAAACTGGGGAACTTGA GTGCAGAAGAGGAGAGTGGAATTCCACGTGTA GTGATAGAGATGGAGGACAC CAGTGGCGAAGGCGACTCTCTGGTCTGTAACT CAGTGGCGAAGGCGACTCTCTGGTCTGTAACT CAGGATTAGATACCCTGGTAGTCCACGCCGTA AACGATGAGTGCTAAGTGTTAGGGGGTTTCCG CCCCTTAGTGCTGCAGCTAACGCATTAAGCACT CCGCCTGGGGAGTACGGTCGCAAGACTGAAAC CCGCCTGGGGAGTACGGTCGCAAGACTGAAAC TCAAAGGAATTGACGGGGGCCCGCACAAGCGG AGAACCTTACCAGGTCTTGACATCCTCTGACA ATCCTAGAGATAGGACGTCCCCTTCGGGGGCA ATCCTAAAAGTOGTGCATGGTTGTCGTCAGCT CGTGTCGTGAGATGTTGGGTTAAGTCCCGCAA CGAGCGCAACCCTTGATCTTAGTTGCCAGCATT CAGTTGGGCACTCTAAGGTGACTGCCGGTGAC AAACCGGAGGAAGGTGGGGATGACGTCAAAT CATCATGCCCCTTATGACCTGGGCTACACACGT GCTACAATGGACAGAACAAAGGGCAGCGAAA CCGCGAGGTTAAGCCAATCCCACAAATCTGTT CTCAGTTCGGATCGCAGTCTGCAACTCGACTGC GTGAAGCTGGAATCGCTAGTAATCGCGGATCA GCATGCCGCGGTGAATACGTTCCCGGGCCTTG TACACACCGCCCGTCACACCACGAGAGTTTGT TACACACCGCCCGTCACACCACGAGAGTTTGT AGCCAGCCGCCGAAGGTGGGACAGATGATTGG GGGGCCACTGGTGTTCCTCCACATCTCTACGCA TTTCACCGCTACACGTGGAATTCCACTCTCCTC TTCACCGCTACACGTGGAATTTCCAATGACO TTCTGCACTCAAGTTCCCCAGTTTCCAATGACE ACTTAAGAAACCGCCTGCGAGCCCTTTACGCC CAATAATTCCGGACAACGCTTGCCACCTACGT ATTACCGCGGCTGCTGGCACGTAGTTAGCCGT AGCTTCTGGTTAGGTACCGTCAAGGTGCCGCC CTATTTGAACGGCACTTGTTCTTCCCTAACAAC AGAGCTTTACGATCCGAAAACCTTCATCACTC ACGCGGCGTTGCTCCGTCAGACTTTCGTCCATT GCGGAAGATTCCCTACTGCTGCCTCCCGTAGG AGTCTGGGCCGTGTCTCAGTCCCAGTGTGGCC AGTCTGGCTCAGGTCGGCTACGCATCGTCGC GATCACCCTCTCAGGTCGGCTACGCATCGTCGC GCGCCGCGGGTCCATCTGTAAGTGGTAGCCGA AGCCACCTTTTATGTCTGAACCATGCGGTTCAA ACAACCATCCGGTATTAGCCCCGGTTTCCCGG ACAACCATCCGGTATTAGCCCCGGTTTCCCGG AGTTATCCCAGTCTTACAGGCAGGTTACCCAC GTGTTACTCACCCGTCCGCCGCTAACATCAGG GTATTAGGCACGCCGCCAGCG

FIGURE 3 - The 16S rRNA sequence generated for isolate-2 had 1600 bases.

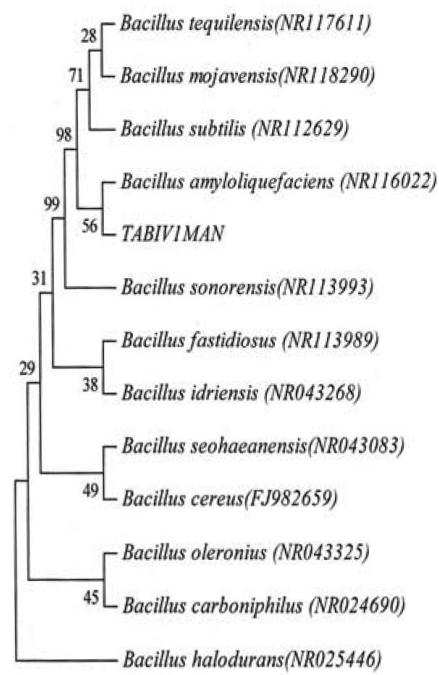

FIGURE 4 - Neighbor-joining tree based on 16S rRNA (1600) sequences showing the relationship between unknown isolate TABSIV1MAN and other closely related species of the genus Bacillus. 
GGGAGGGAGGTGACCGGATTATTGGGCGTAAGGGCT CCGCAGGCGGTTTCTTAAGTCTGATGTGAAAGCCCCC GGCTCAACCGGGGAGGGTCATTGGAAACTGGGGAAC TTGAGTGCAGAAGAGGAGAGTGGAATTCCACGTGTA GCGGTGAAATGCGTAGAGATGTGGAGGAACACCAGT GGCGAAGGCGACTCTCTGGTCTGTAACTGACGCTGA GGAGCGAAAGCGTGGGGAGCGAACAGGATTAGATAC CCTGGTAGTCCACGCCGTAAACGATGAGTGCTAAGT GTTAGGGGGTTTCCGCCCCTTAGTGCTGCAGCTAACG CATTAAGCACTCCGCCTGGGGAGTACGGTCGCAAGA CTGAAACTCAAAGGAATTGACGGGGGCCCGCACAAG CGGTGGAGCATGTGGTTTAATTCGAAGCAACGCGAA GAACCTTACCAGGTCTTGACATCCTCTGACAATCCTA GAGATAGGACGTCCCCTTCGGGGGCAGAGTGACAGG TGGTGCATGGTTGTCGTCAGCTCGTGTCGTGAGATGT TGGGTTAAGTCCCGCAACGAGCGCAACCCTTGATCTT AGTTGCCAGCATTCAGTTGGGCACTCTAAGGTGACTG CCGGTGACAAACCGGAGGAAGGTGGGGATGACGTCA AATCATCATGCCCCTTATGACCTGGGCTACACACGTG CTACAATGGACAGAACAAAGGGCAGCGAAACCGCGA GGTTAAGCCAATCCCACAAATCTGTTCTCAGTTCGGA TCGCAGTCTGCAACTCGACTGCGTGAAGCTGGAATCG CTAGTAATCGCGGATCAGCATGCCGCGGGTGAATAC GTTCCCGGGCCTTGTACACACCGCCCGTCACACCACG AGAGTTTGTAACACCCGAAGTCGGTGAGGTAACCTTT TAGGAGCCAGCCGCCGAAGGTGGGACAGATGATTGG GGGTGAAGTCGTAACAAGGTAGCCGTATCGGAAGGT GCGCTGAACCCCCCCCCTTTCTTATATAAATCCTTTCG CTTCTTTCGCTCCTCAGCGTCAGTTACAGACCAGAGA GTCGCCTTCGCCACTGGTGTTCCTCCACATCTCTACG CATTTCACCGCTACACGTGGAATTCCACTCTCCTCTTC TGCACTCAAGTTCCCCAGTTTCCAATGACCCTCCCCG GTTGAGCCGGGGGCTTTCACATCAGACTTAAGAAAC CGCCTGCGAGCCCTTTACGCCCAATAATTCCGGACAA CGCTTGCCACCTACGTATTACCGCGGCTGCTGGCACG TAGTTAGCCGTGGCTTTCTGGTTAGGTACCGTCAAGG TGCCGCCCTATTTGAACGGCACTTGTTCTTCCCTAAC AACAGAGCTTTACGATCCGAAAACCTTCATCACTCAC GCGGCGTTGCTCCGTCAGACTTTCGTCCATTGCGGAA GATTCCCTACTGCTGCCTCCCGTAGGAGTCTGGGCCG TGTCTCAGTCCCAGTGTGGCCGATCACCCTCTCAGGT CGGCTACGCATCGTCGCCTTGGTGAGCCGTTACCTCA CCAACTAGCTAATGCGCCGCGGGTCCATCTGTAAGTO GTAGCCGAAGCCACCTTTTATGTCTGAACCATGCGGT TCAAACAACCATCCGGTATTAGCCCCGGTTTCCCGGA GTTA TCCCAGTCTTACAGGCAGGTTACCCACGTGTTA CTCACCCGTCCGCCGCTAACATCAGGGAGCAAGCTCC CATCTGTCCGCTCGACTTGCATGTATTAGGCACGCCG CCAGCGTTCGTCCTGAGCGGGGCCAAAAAACCAAAA AAAAAAAAAAAAAACCTTTAACCCT

FIGURE 5 - The 16S rRNA sequence generated for isolate-3 had 1822 bases.

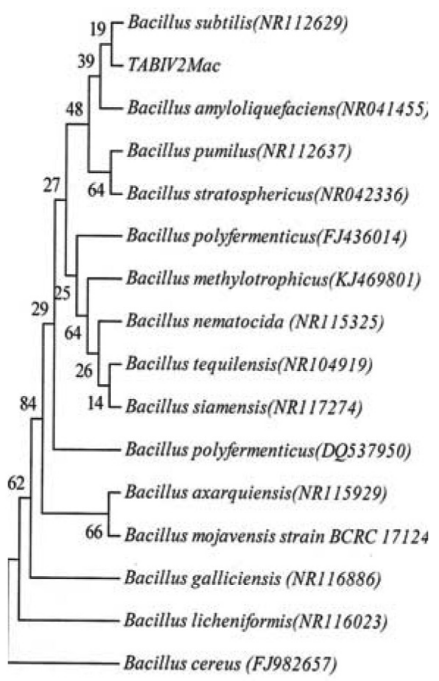

FIGURE 6 - Neighbor-joining tree based on 16S rRNA (1822) sequences showing the relationship between unknown isolate TABSIV2MAC and other closely related species of the genus Bacillus.
TAAGTCTGATGTGAAAGCCCACGGCTCAACCGTGG AGGGTCATTGGAAACTGGAAAACTTGAGTGCAGA AGAGGAAAGTGGAATTCCATGTGTAGCGGTGAAA TGCGCAGAGATATGGAGGAACACCAGTGGCGAAG GCGACTTTCTGGTCTGTAACTGACGCTGATGTGCG AAAGCGTGGGGATCAAACAGGATTAGATACCCTG GTAGTCCACGCCGTAAACGATGAGTGCTAAGTGTT AGGGGGTTTCCGCCCCTTAGTGCTGCAGCTAACGC ATTAAGCACTCCGCCTGGGGAGTACGACCGCAAG GTTGAAACTCAAAGGAATTGACGGGGACCCGCAC AAGCGGTGGAGCATGTGGTTTAATTCGAAGCAAC GCGAAGAACCTTACCAAATCTTGACATCCTTTGAC AACTCTAGAGATAGAGCCTTCCCCTTCGGGGGACA AAGTGACAGGTGGTGCATGGTTGTCGTCAGCTCGT GTCGTGAGATGTTGGGTTAAGTCCCGCAACGAGCG CAACCCTTAAGCTTAGTTGCCATCATTAAGTTGGG CACTCTAAGTTGACTGCCGGTGACAAACCGGAGG AAGGTGGGGATGACGTCAAATCATCATGCCCCTTA TGATTTGGGCTACACACGTGCTACAATGGACAATA CAAAGGGCAGCGAAACCGCGAGGTCAAGCAAATC CCATAAAGTTGTTCTCAGTTCGGATTGTAGTCTGC AACTCGACTACATGAAGCTGGAATCGCTAGTAATC GTAGATCAGCATGCTACGGTGAATACGTTCCCGGG TCTTGTACACACCGCCCGTCACACCACGAGAGTTT GTAACACCCGAAGCCGGTGGAGTAACCATTTGGA GCTAGCCGTCGAAGGTGGGACAAATCCACTGGTG TTCCTCCATATCTCTGCGCATTTCACCGCTACACAT GGAATTCCACTTTCCTCTTCTGCACTCAAGTTTTCC AGTTTCCAATGACCCTCCACGGTTGAGCCGTGGGC TTTCACATCAGACTTAAAAAACCGCCTACGCGCGC TTTACGCCCAATAATTCCGGATAACGCTTGCCACC TACGTATTACCGCGGCTGCTGGCACGTAGTTAGCC GTGGCTTTCTGATTAGGTACCGTCAAGACGTGCAT AGTTACTTACACGTATGTTCTTCCCTAATAACAGA GTTTTACGATCCGAAGACCTTCATCACTCACGCGG CGTTGCTCCGTCAGGCTTTCGCCCATTGCGGAAGA TTCCCTACTGCTGCCTCCCGTAGGAGTCTGGACCG TGTCTCAGTTCCAGTGTGGCCGATCACCCTCTCAG GTCGGCTACGTATCGTCGCCTTGGTAAGCCGTTAC CTTACCAACTAGCTAATACGGCGCGGGTCCATCTA TAAGTGATAGCAAAACCATCTTTCACTATCGAACC ATGCGGTTCGAAATATTATCCGGTATTAGCTCCGG TTTCCCGAAGTTATCCCAGTCTTATAGGTAGGTTA CCCACGTGTTACTCACCCGTCCGCCGCTAACGTCA AAGGAGCAAGCTCCTTGTCTGTTCGCTCGACTTGC ATGTATTAGGCACGCCGCCAGCGT

FIGURE 7 - The 16S rRNA sequence generated for isolate-4 had 1590 bases.

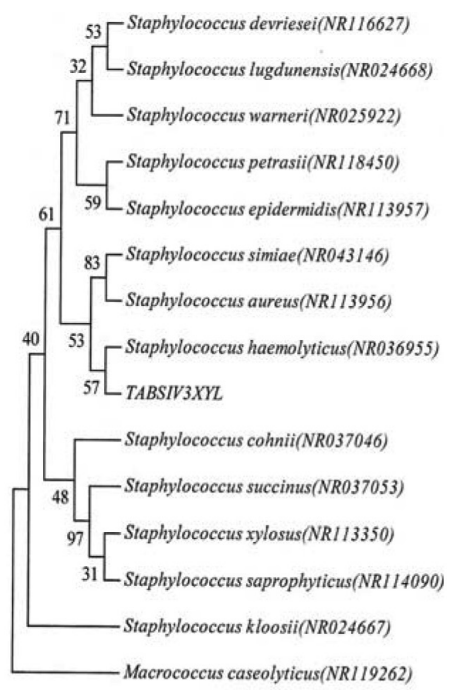

FIGURE 8 - Neighbor-joining tree based on 16S rRNA (1590) sequences showing the relationship between unknown isolate TABSIV3XYL and other closely related species of the genus Staphylococcus. 
CTTAAGTCTGATGTGAAAGCCCCCGGCTCAACCGGG GAGGGTCATTGGAAACTGGGGAACTTGAGTGCAGAA GAGGAGAGTGGAATTCCACGTGTAGCGGTGAAATGC GTAGAGATGTGGAGGAACACCAGTGGCGAAGGCGAC TCTCTGGTCTGTAACTGACGCTGAGGAGCGAAAGCGT GGGGAGCGAACAGGATTAGATACCCTGGTAGTCCAC GCCGTAAACGATGAGTGCTAAGTGTTAGGGGGTTTCC GCCCCTTAGTGCTGCAGCTAACGCATTAAGCACTCCG CCTGGGGAGTACGGTCGCAAGACTGAAACTCAAAGG AATTGACGGGGGCCCGCACAAGCGGTGGAGCATGTG GTTTAATTCGAAGCAACGCGAAGAACCTTACCAGGT CTTGACATCCTCTGACAATCCTAGAGATAGGACGTCC CCTTCGGGGGCAGAGTGACAGGTGGTGCATGGTTGT CGTCAGCTCGTGTCGTGAGATGTTGGGTTAAGTCCCG CAACGAGCGCAACCCTTGATCTTAGTTGCCAGCATTC AGTTGGGCACTCTAAGGTGACTGCCGGTGACAAACC GGAGGAAGGTGGGGATGACGTCAAATCATCATGCCC CTTATGACCTGGGCTACACACGTGCTACAATGGGCAG AACAAAGGGCAGCGAAACCGCGAGGTTAAGCCAATC CCACAAATCTGTTCTCAGTTCGGATCGCAGTCTGCAA CTCGACTGCGTGAAGCTGGAATCGCTAGTAATCGCG GATCAGCATGCCGCCGGTGAATACGTTCCCGGGCCTT GTACACACCGCCCGTCACACCACGAGAGTTTGTAAC ACCCGAAGTCGGTGCCACTGGTGTTCCTCCACATCTC TACGCATTTCACCGCTACACGTGGAATTCCACTCTCC TCTTCTGCACTCAAGTTCCCCAGTTTCCAATGACCCTC CCCGGTTGAGCCGGGGGCTTTCACATCAGACTTAAGA AACCGCCTGCGAGCCCTTTACGCCCAATAATTCCGGA CAACGCTTGCCACCTACGTATTACCGCGGCTGCTGGC ACGTAGTTAGCCGTGGCTTTCTGGTTAGGTACCGTCA AGGTGCCGCCCTATTTGAACGGCACTTGTTCTTCCCT AACAACAGAGCTTTACGATCCGAAAACCTTCATCACT CACGCGGCGTTGCTCCGTCAGACTTTCGTCCATTGCG GAAGATTCCCTACTGCTGCCTCCCGTAGGAGTCTGGG CCGTGTCTCAGTCCCAGTGTGGCCGATCACCCTCTCA GGTCGGCTACGCATCGTCGCCTTGGTGAGCCGTTACC TCACCAACTAGCTAATGCGCCGCGGGTCCATCTGTAA GTGGTAGCCGAAGCCACCTTTTATGTCTGAACCATGC GGTTCAAACAACCATCCGGTATTAGCCCCGGTTTCCC GGAGTTATCCCAGTCTTACAGGCAGGTTACCCACGTG TTACTCACCCGTCCGCCGCTAACATCAGGGAGCAAGC TCCCATCTGTCCGCTCGACTTGCATGTATTAGGCACG CCGCCAGCG

FIGURE 9 - The 16S rRNA sequence generated for isolate-5 had 1550 bases.

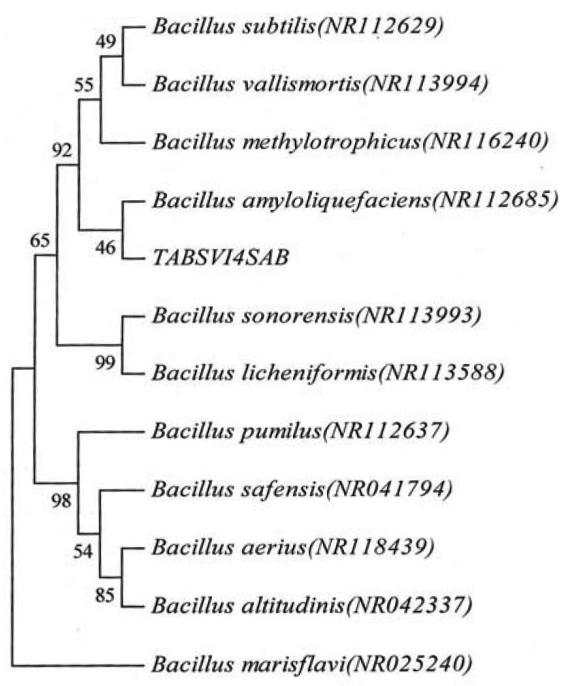

FIGURE 10 - Neighbor-joining tree based on 16S rRNA (1550) sequences showing the relationship between unknown isolate TABSIV4SAB) and other closely related species of the genus Bacillus.
TABLE II - Microbial isolates from non-parenterals identified by 16S rRNA sequence

\begin{tabular}{lcc}
\hline Isolates & Fprmulation & $\begin{array}{c}\text { Microbial isolate } \\
\text { (contaminant) identified by } \\
\text { 16S rRNA sequence }\end{array}$ \\
\hline Isolates-1 & Syrup & Bacillus tequilensis \\
Isolates-2 & Tablets & Bacillus amyloliquefaciens \\
Isolates-3 & Tablets & Bacilus subtilis \\
Isolates-4 & Tablets & Sthaphylococcus \\
Isolates-5 & Tablets & Bacillus amyloliquefaciens \\
\hline
\end{tabular}

other microorganisms of interest (Jimenez, Ignar, 2000). Although it takes 6 to 7 hours more than PCR methods, 16S rRNA sequence-based analysis gives very accurate information about all microbial contaminants and also confirms the presence of any other pathogens by virtue of its specificity (Rompre et al., 2002; Gee et al., 2003; Rhoads et al., 2012). As discussed earlier about sequence analysis, this study also confirmed $16 \mathrm{~S}$ rRNA as the most ideal and suitable method for the identification of microbial contaminants in non-parenteral products. Hence, microbial quality control for non-parenteral products by 16S rRNA sequence-based identification can be employed on a routine basis as a pharmacopoeial protocol to ensure simplicity, reliability and rapidity.

\section{ACKNOWLEDGMENT}

The authors acknowledge the University Grants Commission, New Delhi for the grant under the Major Research Project. Dr. A. Leyva helped with English editing of the manuscript.

\section{REFERENCES}

BANSAL, A. K.; MEYER, T.E. Evolutionary analysis by whole genome comparisons. J. Bacteriol., v.184, n.8, p.2260-2272, 2002.

COOK, V.J.; TURENNE, C.Y.; WOLFE, J.; PAULS, R.; KABANI, A. Conventional methods versus $16 \mathrm{~S}$ ribosomal DNA sequencing for identification of nontuberculous Mycobacterium: cost analysis. J. Clin. Microbiol., v.41, n.3, p.1010-1015, 2003. 
DEVEREUX, R.; WILKINSON, S.S. Amplification of ribosomal RNA sequences. In: KOWALCHUK, G.A. et al (Eds.). Molecular microbial ecology manual. 2. ed. Dordrecht; London: Kluwer Academic Publishers, 2004. v.3.01, p.509-522.

EASTER, C.M. Rapid microbiological methods in the pharmaceutical industry. Denver: Interpharm CRC Press, 2003. p.161-177.

GEE, J.E.; SACCHI, C.T.; GLASS, M.B.; DE, B.K.; WEYANT, R.S.; LEVETT, P.N.; WHITNEY, A.M.; HOFFMASTER, A.R.; POPOVIC, T. Use of $16 \mathrm{~S}$ rRNA gene sequencing for rapid identification and differentiation of Burkholderia pseudomallei and B. mallei.J. Clin. Microbiol., v.41, n.10, p.4647-4654, 2003.

GENBANK. GenBank Database. Available at: < http://www. ncbi.nlm.nih.gov/genbank/>. Accessed on: 2014

GHYSELINCK, J.; PFEIFFER, S.; HEYLEN, K.; SESSITSCH, A.; DE VOS, P. The Effect of Primer Choice and Short Read Sequences on the Outcome of 16S rRNA Gene Based Diversity Studies. Plos One, v.8, p.1-14, 2013.

HALL, L.; DOERR, K.A.; WOHLFIEL, S.L.; ROBERTS, G.D. Evaluation of the MicroSeq system for identification of Mycobacteria by $16 \mathrm{~S}$ ribosomal DNA sequencing and its integration into a routine clinical mycobacteriology laboratory. J. Clin. Microbiol., v.41, n.4, p.1447-1453, 2003.

INDIAN PHARMACOPOEIA. Microbiological examination of Non-sterile products: Tests for specified microorganisms. The Indian Pharmacopoeia commission Central Indian pharmacopoeia laboratory Government of India, Ministry of health \& family welfare, Ghaziabad, v.1, p.36-45, 2007.

JASSON, V.; JACXSENS, L.; LUNING, P.; RAJKOVIC, A.; UYTTENDAELE, M. Alternative microbial methods: An overview and selection criteria. Food Microbiol., v.27, n.6, p.710-730, 2010.

JIMENEZ, L.; RAYMOND IGNAR, S.S. Use of PCR analysis for detecting low levels of bacteria and mold contamination in pharmaceutical samples. J. Microbiol. Meth., v.41, n.3, p.259-265, 2000.

LANE, D.J. 16S/23S rRNA sequencing. In: STACKEBRANDT, E.; GOODFELLOW, M., (Eds.). Nucleic acid techniques in bacterial systematics. New York: John Wiley and Sons, 1991. p. 115-175.
LANE, D.J.; PACE, B.; OLSEN, G.J.; STAHLT, D.A.; SOGINT, M.; PACE, N.R. Rapid determination of $16 \mathrm{~S}$ ribosomal RNA sequences for phylogenetic analyses. Proc. Natl. Acad. Sci. USA, v.82, p.6955-6959, 1985.

LOEFFLER, J.; HEBERT, H.; SCHUMACHER, U.; REITZE, H.; EINSELE, H. Extraction of fungal DNA from cultures and blood using the QIAamp Tissue Kit. Qiagen News., v.4, p.16-17, 1996.

NEWBY, P. Rapid methods for enumeration and identification in microbiology. In: BAIRD, R.M.; HODGES, N.A.; DENVER, S.P. (Eds.). Handbook of microbiological control: pharmaceuticals and medical devices. London: Taylor \& Francis, 2000. p.107-119.

NIKUNJKUMAR, B.D. Molecular identification of bacteria using 16s rDNA sequencing. Gujarat: Gujarat University, 2012. p.1-62.

NISHIGUCHI,M.K.; DOUKAKIS,P.; EGAN,M.; KIZIRIAN,D.; PHILLIPS,A.; PRENDINI,L.; ROSENBAUM, H.C.; TORRES,E.; WYNER,Y.; DESALLE,R.; GIRIBET,G. DNA Isolation Procedures. In: DE SALLE, R.; GIRIBET, G.; WHEELER, W.C. (eds.). Methods and tools in biosciences and medicine and techniques in molecular systematics and evolution. Switzerland: Birkhauser Verlag Basel, 2002. p.250-287.

PATEL, J.B. 16S rRNA gene sequencing for bacterial pathogen identification in the clinical laboratory. Mol. Diagn., v.6, n.4, p.313-321, 2001.

RHOADS, D.D.; WOLCOTT, R.D.; SUN, Y.; DOWD, S.E. Comparison of culture and molecular identification of bacteria in chronic wounds. Int. J. Mol. Sci., v.13, n.3, p.2535-2550, 2012.

ROMPRE, A.; SERVAIS, P.; BAUDART, J.; DE-ROUBIN, M.R.; LAURENT, P. Detection and enumeration of coliforms in drinking water: current methods and emerging approaches. J. Microbiol. Meth., v.49, n.1, p.31-54, 2002.

ROSA, M.C.; MEDINA, M.R.; VIVAR, M. Microbiological quality of pharmaceutical raw materials. Pharm. Acta Helv., v.70, n.3, p.227-232, 1995.

SENTHILRAJ, R.; PRASAD, G.S.; JANAKIRAMAN, K. Development of microbial quality control protocols for non parenteral products. Pharm. Sci. Monitor, v.5, n.3, p.55-70, 2014. 
SILVA, M.A.C.; CAVALETT, A.; SPINNER, A.; ROSA, D.C.; JASPER, R.B.; QUECINE, M.C.; BONATELLI, M.L.; PIZZIRANI-KLEINER, A.; CORCAO, G.; DE SOUZA LIMA, A.O. Phylogenetic identification of marine bacteria isolated from deep-sea sediments of the eastern South Atlantic Ocean. Springer Plus, v.2, p.127, 2013.

SONG, J.; LEE S.C.; KANG, J.W.; BAEK H.J.; SUH, J.W. Phylogenetic analysis of Streptomyces spp. isolated from potato scab lesions in Korea on the basis of 16S rRNA gene and 16S-23S rDNA internally transcribed spacer sequences. Int. J. Syst. Evol. Microbiol., v.54, pt.1, p.203-209, 2004.

TAMURA, K.; PETERSON, D.; PETERSON, N.; STECHER, G.; NEI, M.; KUMAR, S. MEGA5: molecular evolutionary genetics analysis using maximum likelihood, evolutionary distance, and maximum parsimony. Methods Mol. Biol. Evol., v.28, n.10, p.2731-2739, 2011.

TURNER, S.; PRYER, K.M.; MIAO, V.P.W.; PALMER, J.D. Investigating deep phylogenetic relationships among cyanobacteria and plastids by small subunit rRNA sequence analysis. J. Eukaryot. Microbiol., v.46, n.4, p.327-338, 1999.
UNITED STATES PHARMACOPOEIA. USP. Microbiological examination of non-sterile products: Tests for specified microorganisms. 31. ed. Twinbrook, Parkway Rockvalie: United States Pharmacopoeial Convention, 2012. v.1, p.56-65.

VURAL, H.C.; OZGUN, D. An improving DNA isolation method for identification of anaerobic bacteria in human colostrum and faeces samples. J. Med. Genet. Genom., v.3, n.5, p.95-100, 2011.

WOO, P.C.; NG, K.H.; LAU, S.K.; YIP K.T.; FUNGAM.; LEUNG, K.W.;TAM D.M.; QUE T.L.; YUEN K.Y. Usefulness of the MicroSeq 500 16S ribosomal DNA-based bacterial identification system for identification of clinically significant bacterial isolates with ambiguous biochemical profiles. J. Clin. Microbiol., v.41, n.5, p.1996-2001, 2003.

Received for publication on $21^{\text {th }}$ December 2014 Accepted for publication on $02^{\text {nd }}$ May 2016 